\title{
miR-205 regulates the proliferation and invasion of ovarian cancer cells via suppressing PTEN/SMAD4 expression
}

\author{
PING CHU ${ }^{1}$, AIHUA LIANG ${ }^{1}$, AILI JIANG ${ }^{1}$ and $\mathrm{LU} \mathrm{ZONG}^{2}$ \\ ${ }^{1}$ Department of Gynecology, Yantai Affiliated Hospital of Binzhou Medical University, Yantai, Shandong 264100; \\ ${ }^{2}$ Department of Gynecology and Obstetrics, The First Affiliated Hospital of Xi'an Jiaotong University, \\ Xi'an, Shaanxi 710061, P.R. China
}

Received January 4, 2017; Accepted November 16, 2017

DOI: $10.3892 / \mathrm{ol} .2018 .8313$

\begin{abstract}
MicroRNAs (miRNAs/miRs) are non-coding RNAs that post-transcriptionally control target genes, and are involved in tumorigenesis, apoptosis, proliferation, invasion, metastasis and chemoresistance. However, data concerning miRNAs in ovarian cancer remain incomplete. The present study aimed to identify miRNAs that affected the malignant phenotype of ovarian cancer, and to analyze their potential mechanisms. The data demonstrated that miR-205 promoted cell proliferation and invasion of ovarian cancer cells via suppressing Phosphatase and tensin homolog (PTEN)/mothers against decapentaplegic homolog 4 (SMAD4) expression. Based on the Cancer Genome Atlas database analysis results, it was identified that miR-205 was significantly upregulated in ovarian cancer tissues and markedly correlated with poor prognosis in patients with ovarian cancer; its abnormal expression was also confirmed in tissues from patients with ovarian cancer by reverse transcription quantitative polymerase chain reaction. Additional Gene Ontology analysis revealed that the target genes of miR-205 were associated with cell proliferation and invasion. Consistent with the database analysis, miR-205 overexpression significantly promoted ovarian cancer cell proliferation and invasion in vitro. To additionally explore the mechanism by which miR-205 was associated with proliferation and invasion of ovarian cancer cells, a protein-protein interaction network was constructed based on miR-205 target genes associated with proliferation and invasion, and it was revealed that PTEN and SMAD4 were key
\end{abstract}

Correspondence to: Dr Lu Zong, Department of Gynecology and Obstetrics, The First Affiliated Hospital of Xi'an Jiaotong University, 277 Yanta Xi Road, Xi'an, Shaanxi 710061, P.R. China E-mail: 444517237@qq.com

Abbreviations: PTEN, phosphatase and tensin homolog; SMAD4, mothers against decapentaplegic homolog 4; MMP-2, matrix metalloproteinase-2; MMP-9, matrix metalloproteinase-9

Key words: microRNA-205, ovarian cancer, phosphatase and tensin homolog, mothers against decapentaplegic homolog 4, proliferation, invasion target genes of miR-205. In ovarian cancer tissues, the expression levels of PTEN and SMAD4 were significantly downregulated, suggesting that miR-205 may suppress the expression of PTEN and SMAD4 in vivo. In vitro, miR-205 overexpression markedly suppressed the expression of SMAD4 and PTEN, additionally verifying that PTEN and SMAD4 were the target genes of miR-205 in ovarian cancer cells. These results elucidated the tumor-promoting role of miR-205 and established miR-205 as a potential treatment target for ovarian cancer.

\section{Introduction}

Ovarian cancer is one of the most common and lethal types of cancer, it is the seventh most common cancer in women and the eighth most common cause of recent global cancer mortality, with 5 -year survival rates of $<45 \%$ (1). A growing number of treatment targets associated with ovarian cancer were identified, but knowledge concerning the pathogenesis is limited (2).

microRNAs (miRNAs), a class of small non-coding RNA molecules, regulate gene expression by interacting with the 3'-untranslated regions of target genes and serve a significant role in the carcinogenesis or prognosis prediction in various types of tumor $(3,4)$. miRNAs are involved in numerous biological process by regulating the expression level of target genes, such as cell proliferation, invasion, and migration (4). Conversely, miRNA may serve as a relatively independent objective indices that reflect the biological behaviors of tumors, and may be of auxiliary value in the analysis of prognostic factors of various tumors (2). Therefore, deregulated miRNAs may provide a novel understanding of the underlying mechanisms of tumor progression and prognosis prediction.

During ovarian cancer occurrence and progression, the abnormal expression of miRNAs was also identified to exert important functions: miR-298, miR-595, miR-136 and miR-222-3p were downregulated in ovarian cancer tissue, and associated with tumor growth, invasion and metastasis (5-8). In contrast, miR-224, miR-194 and miR-572 were upregulated in ovarian cancer tissues (9-11). Although these miRNAs were also suggested to be involved in the development of ovarian cancer, knowledge concerning how miRNAs to affect the occurrence and progression of ovarian cancer, particularly for their function in prognosis prediction, remains rare. Therefore, 
there is a requirement for therapeutic targets to improve the outcomes of patients with ovarian cancer.

In the present study, it was identified that miR-205 was significantly upregulated in ovarian cancer tissues. High levels of miR-205 were associated with cell proliferation and invasion. miR-205 exerted these functions by primarily targeting mothers against decapentaplegic homolog 4 (SMAD4) and Phosphatase and tensin homolog (PTEN). These results assist to elucidate the pathogenesis of ovarian cancer.

\section{Materials and methods}

Database analysis. The Cancer Genome Atlas (TCGA) data concerning miRNA expression levels and clinical data in patients with all types of ovarian cancer were obtained from https:/cancergenome.nih.gov/; November 6th, 2016). The target genes of miR-205 were obtained from miRTarBase database (http://mirtarbase.mbc.nctu.edu.tw/php/index.php, November 6th, 2016) (4). Cytoscape software (version 3.4.0; http://www.cytoscape.org/) was used to map the Gene Ontology (GO) of 28 target genes of miR-205. The Search Tool for the Retrieval of Interacting Genes/Proteins database was used to search for known or predicted protein interactions of these target genes (search terms were all 28 target genes symbols), that may aid in the comprehensive description of cellular mechanisms and functions (http://string-db.org/; November 6th, 2016) (12). According to a keyword earch (PTEN and SMAD4) on Human Protein Atlas website (www.proteinatlas. org; November 6th, 2016), IHC data of ovarian cancer related to PTEN and SMAD4 protein expression were downloaded.

Patient tissues. Surgical resection specimens of 10 ovarian cancer cases (mean age, 45.87 \pm 3.23 ) and adjacent normal ovary tissues were collected via resection between June 2015 and July 2016 in the Yantai Affiliated Hospital of Binzhou Medical University (Yantai, China), and were preserved in liquid nitrogen. The present study was approved by the Research Ethics Boards of Binzhou Medical University and Yantai Affiliated Hospital. Written informed consent was obtained from each patient.

Cell culture and miRNA transfection. The human ovarian cancer OVCAR-3 cell line was purchased from the Cell Resource Center of Peking Union Medical College (Beijing, China) and was incubated at $37^{\circ} \mathrm{C}$ in a humidified atmosphere containing 5\% $\mathrm{CO}_{2}$. OVCAR-3 cells were maintained in high-glucose Dulbecco's modified Eagle's medium (DMEM; Gibco; Thermo Fisher Scientific, Inc., Waltham, MA USA), supplemented with $10 \%$ fetal bovine serum (FBS; Thermo Fisher Scientific, Inc.), $100 \mathrm{U} / \mathrm{ml}$ penicillin and $100 \mu \mathrm{g} / \mathrm{ml}$ streptomycin (Thermo Fisher Scientific, Inc.). miR-205 mimics and negative control (NC) sequences were designed and synthesized by Guangzhou RiboBio Co., Ltd. (Guangzhou, China). The miR-205 mimics and negative control sequence were transfected into the OVCAR-3 cell line using Lipofectamine ${ }^{\circledR}$ 2000 (Invitrogen; Thermo Fisher Scientific, Inc.), according to the manufacturer's protocol. Following $48 \mathrm{~h}$ transfection, cells were used for the subsequent experiments.

Cell counting kit-8 (CCK-8) and anchorage-independent growth assay. miR-205-transfected OVCAR-3 cells, negative control sequence transfected OVCAR-3 cells and untransfected OVCAR-3 cells were seeded into 96-well plates at a density of $5 \times 10^{3}$ cells/well in triplicate and cultivated at $37^{\circ} \mathrm{C}$ in an incubator with $5 \% \mathrm{CO}_{2}$. CCK-8 $(10 \mu \mathrm{l}$; Sigma-Aldrich; Merck KGaA; Darmstadt, Germany) was added to each well every $24 \mathrm{~h}$. Subsequently, the absorbance of the cells was measured at $450 \mathrm{~nm}$ using a microplate reader. For the anchorage-independent growth assay, soft agar (Sigma-Aldrich; Merck $\mathrm{KGaA}$ ) at a concentration of $0.8 \%$ in DMEM culture media was placed into 6-well plates and allowed to solidify. A top layer was then placed into each well containing $1 \times 10^{4}$ cells in $0.48 \%$ agar in DMEM culture media and allowed to solidify, then $1 \mathrm{ml}$ DMEM culture media was placed on top of the solidified agar. Plates were incubated at $37^{\circ} \mathrm{C}$ for 2 weeks, the media was removed and the cells (OVCAR-3 cells transfected with miR-205 mimics and NC sequence, untransfected OVCAR-3 cells) were fixed in $10 \%$ buffered formalin containing $0.5 \%$ crystal violet at $25^{\circ} \mathrm{C}$ for $2 \mathrm{~h}$. Colonies were counted and analyzed using the Prism software (version 6.00, La Jolla California USA) (13).

Gelatin zymography assay. Activities of matrix metalloproteinase (MMP)-2 and MMP-9 were assessed by a gelatin zymography assay. OVCAR-3 cells were cultured at $37^{\circ} \mathrm{C}$ in a humidified atmosphere containing $5 \% \mathrm{CO}_{2}$ for $24 \mathrm{~h}$ following transfection with miR-205 and control sequences. At the end of the incubation, $30 \mu \mathrm{l}$ culture supernatant was mixed with sample buffer and resolved on a $10 \%$ gel using SDS-PAGE, without heat denaturation. The gel was co-polymerized and contained $0.5 \mathrm{mg} / \mathrm{ml}$ gelatin (Sigma-Aldrich; Merck KGaA). The gel was washed twice for 30 min with renaturation buffer $(2.5 \%$ Triton $\mathrm{X}-100)$ at room temperature prior to incubation in the incubation buffer $(50 \mathrm{mM}$ Tris- $\mathrm{HCl} \mathrm{pH} 7.5,200 \mathrm{mM} \mathrm{NaCl}$, $10 \mathrm{mM} \mathrm{CaCl} 2,1 \mu \mathrm{M} \mathrm{ZnCl} 2)$ at $37^{\circ} \mathrm{C}$ for $16 \mathrm{~h}$. Subsequently, the gel was stained for $2 \mathrm{~h}$ in $0.25 \%$ at $25^{\circ} \mathrm{C}$. Coomassie Brilliant Blue R-250 (Thermo Fisher Scientific, Inc.), and then de-stained (30\% methanol, $10 \%$ glacial acetic acid, $60 \%$ distilled water) at $25^{\circ} \mathrm{C}$ for $30 \mathrm{~min}$. White bands were observed using a ChemiDoc MP Imaging System (Bio-Rad Laboratories, Inc., Hercules, CA, USA) against a blue background following de-staining, indicating the gelatinolytic activities of MMP-2 and MMP-9.

Western blotting. miR-205-transfected cells and control cells were collected using centrigugation $(\mathrm{x} 1,000 \mathrm{~g})$ for $5 \mathrm{~min}$ at $25^{\circ} \mathrm{C}$ and lysed in lysis buffer (Beijing CoWin Biotech Co., Ltd., Beijing, China) in the presence of protease inhibitors for $30 \mathrm{~min}$ to extract the total protein. Bicinchoninic acid assays (Beijing CoWin Biotech) were used to quantify protein levels. Subsequently, $30 \mu \mathrm{g}$ protein from each sample was loaded onto $10 \%$ SDS-PAGE gels (ready-made SDS-PAGE gels was purchased from Beijing CoWin Biotech). and subjected to gel electrophoresis. Proteins were then transferred to nitrocellulose membranes (Sigma-Aldrich; Merck $\mathrm{KGaA}$ ), which were then blocked at $25^{\circ} \mathrm{C}$ with $3 \%$ bovine serum albumin blocking buffer (Invitrogen; Thermo Fisher Scientific, Inc.) for $1 \mathrm{~h}$. Membranes were then incubated with primary rabbit antibodies targeting SMAD4 (1:2,000 dilution; cat no. ab40759; Abcam, Cambridge, UK), PTEN (1:1,000 dilution; cat no. ab32199; Abcam), or GAPDH 
(1:5,000 dilution; cat no. ab70699; Abcam) overnight at $4{ }^{\circ} \mathrm{C}$, followed by incubation with goat anti-rabbit horseradish peroxidase-conjugated secondary antibody (1:3,000 dilution; cat no. CW0103; Beijing CoWin Biotech) for $1 \mathrm{~h}$ at room temperature. Detection was facilitated using an enhanced chemiluminescence kit (Beijing CoWin Biotech Co., Ltd.) and images were analyzed using Quantity One software (version 4.6.9, Bio-Rad Laboratories, Inc.).

$R N A$ extraction and reverse transcription quantitative polymerase chain reaction ( $R T-q P C R)$. Resected tumor tissues from patients with ovarian cancer were harvested, and total endogenous RNA was isolated using the RNeasy Mini kit (Qiagen GmbH, Hilden, Germany) following the manufacturer's protocol. RNA concentration was measured and its quality was evaluated by absorption ratio at 260/280 nm using NanoDrop 2000 (NanoDrop Technologies; Thermo Fisher Scientific, Inc., Pittsburgh, PA, USA). A total of 10 ng RNA from each sample was reverse transcribed in triplicate using Step One Plus Real-Time PCR kit (Applied Biosystems; Thermo Fisher Scientific, Inc.) according to the manufacturer's protocol. The TaqMan microRNA Assay kit (Applied Biosystems; Thermo Fisher Scientific, Inc.) was used to examine the expression of miR-205 in ovarian cancer and normal tissues according to manufacturer's protocol. The relative expression levels of miRNAs were analyzed using the $\mathrm{Cq}$ method (14) and were normalized against U6 expression. miR-205, 5'-CTTGTCCTTCAT TCCACCGGA-3' (forward) and 5'-TGCCGCCTGAAC TTCACTCC-3' (reverse). U6, 5'-CTCGCTTCGGCAGCA CA-3' (forward) and 5'-AACGCTTCACGAATTTGCGT-3' (reverse). Reaction conditions: $95^{\circ} \mathrm{C}, 5 \mathrm{~min} ; 95^{\circ} \mathrm{C}, 10 \mathrm{sec}$, $60^{\circ} \mathrm{C}, 20 \mathrm{sec}, 40$ cycles.

Cell invasion. Transwell assays were used to determine the abilities of cell invasion. Briefly, $20 \mu \mathrm{g}$ Matrigel ${ }^{\circledR}$ was coated in the upper chambers, then $600 \mu 1$ DMEM containing $10 \%$ FBS was added to the lower chambers. A total of $1 \times 10^{5}$ cells (OVCAR-3 cells transfected with miR-205 mimics and NC sequence, untransfected OVCAR-3 cells) suspended in $100 \mu \mathrm{l}$ $1 \%$ serum-free medium were added to the upper chambers and cultured at $37^{\circ} \mathrm{C}$ for $24 \mathrm{~h}$. Finally, the cells that traversed the membrane were stained with crystal violet $(0.1 \%)$ at $27^{\circ} \mathrm{C}$, five random fields were counted using light microscopy (magnification, $\mathrm{x} 400$ ).

Statistical analysis. Statistical analyses were performed using the SPSS 21.0 (IBM Corp, Armonk, NY, USA) and GraphPad Prism 5 (GraphPad Software Inc., La Jolla, CA, USA). TCGA data for paired samples were analyzed by a paired sample t-test. The log-rank test for the generated Kaplan-Meier curve was conducted to evaluate the association between the expression level of miR-205 and the survival rate. Growth curves, anchorage-independent growth and Transwell data were analyzed using a one-way repeated-measures analysis of variance with Fisher's least significant difference post-hoc tests. Immunohistochemical (IHC) data for the unpaired samples were analyzed by an independent samples t-test. All experiments were repeated 3 times, and $\mathrm{P}<0.05$ was considered to indicate a statistically significant difference.
Table I. Top 20 significantly upregulated microRNAs in ovarian cancer tissues.

\begin{tabular}{|c|c|c|c|}
\hline No. & Name & $\begin{array}{c}\log _{2} \\
\text { (T/N fold change) }\end{array}$ & P-value \\
\hline 1 & hsa-mir-141 & 5.053592 & 0.00000013 \\
\hline 2 & hsa-mir-200c & 4.379344 & 0.00000157 \\
\hline 3 & hsa-mir-96 & 5.530791 & 0.00000676 \\
\hline 4 & hsa-mir-183 & 5.473592 & 0.00003524 \\
\hline 5 & hsa-mir-130b & 3.094745 & 0.00019624 \\
\hline 6 & hsa-mir-182 & 4.469734 & 0.00023611 \\
\hline 7 & hsa-mir-200a & 3.955599 & 0.00025332 \\
\hline 8 & hsa-mir-3934 & 3.681847 & 0.00027821 \\
\hline 9 & hsa-mir-15b & 2.553105 & 0.00033210 \\
\hline 10 & hsa-mir-429 & 4.095076 & 0.00039806 \\
\hline 11 & hsa-mir-1976 & 2.411089 & 0.00051727 \\
\hline 12 & hsa-mir-205 & 7.758691 & 0.00064276 \\
\hline 13 & hsa-mir-106b & 1.823214 & 0.00067700 \\
\hline 14 & hsa-mir-3613 & 2.627981 & 0.00071648 \\
\hline 15 & hsa-mir-942 & 2.608719 & 0.00083393 \\
\hline 16 & hsa-mir-210 & 4.852877 & 0.00085726 \\
\hline 17 & hsa-mir-1307 & 2.082525 & 0.00099215 \\
\hline 18 & hsa-mir-32 & 2.176342 & 0.00113742 \\
\hline 19 & hsa-mir-142 & 3.553646 & 0.00193837 \\
\hline 20 & hsa-mir-16-2 & 2.122048 & 0.00010411 \\
\hline
\end{tabular}

$\mathrm{T} / \mathrm{N}$, relative expression level of tumor tissues/relative expression level of normal tissues; miR, microRNA.

\section{Results}

miR-205 is upregulated in ovarian cancer tissues and associated with poor prognosis. To identify the novel differentially expressed miRNAs associated with ovarian cancer occurrence and development, a TCGA_ miRNA dataset was used in the present study. A total of 108 differentially-expressed miRNAs (Fig. 1A; P<0.01) were identified, and the first 20 upregulated miRNAs with significant differences are summarized in Table I. Of these, miR-205 was upregulated in 308 tissues from patients with ovarian cancer (Fig. $1 \mathrm{~B} ; \mathrm{P}=0.028$ ); it is a rarely-identified miRNA in ovarian cancer and was therefore of interest. To additionally validate the abnormally upregulated expression level of miR-205, 10 pairs of ovarian cancer tissue specimens from clinical samples were compared. Consistent with the results from TCGA, miR-205 was significantly upregulated in the tumor tissue specimens compared with matched normal controls (Fig. $1 C ; \mathrm{P}=0.0076$ ). Subsequently, the prognosis-predictive role of miR-205 expression level as a prognostic marker was investigated. First, the miR-205 expression values of 308 patients with ovarian cancer who underwent RNA-seq analysis from the TCGA_ miRNA dataset were arrayed in ascending order of RNA-seq value, and the median value was $34251.63 \mathrm{reads} / \mathrm{kilobase} / \mathrm{million}$ mapped reads (RPKM). Then, the patients were divided according to the median value $(34,251.63$ RPKM) into miR-205 high expression $(>34,251.63)$ and miR-205 low $(<34,251.63)$ expression 

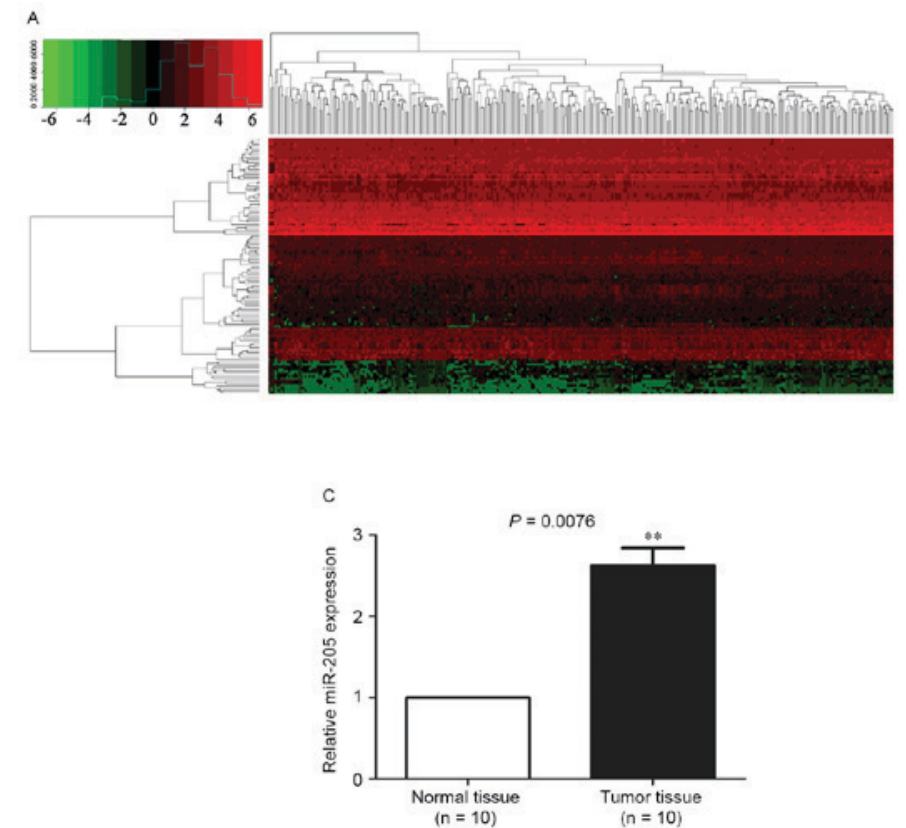

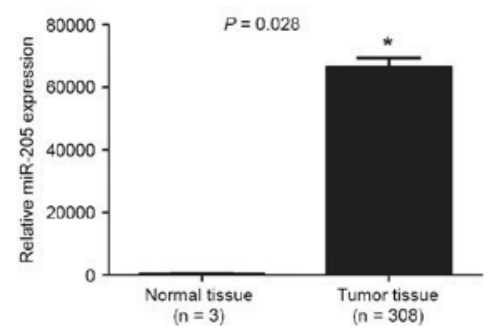

D

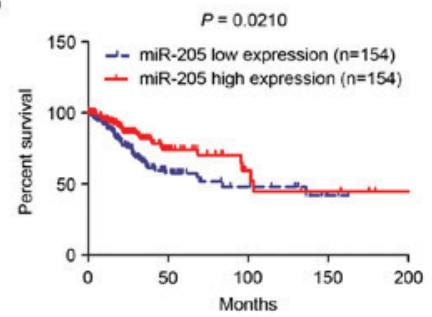

Figure 1. miR-205 is upregulated in ovarian cancer and correlated with poor prognosis. (A) The differential expression of miRNAs from TCGA database are presented by heat map. (B) miR-205 expression was upregulated in ovarian cancer tissues compared with adjacent normal tissues from TCGA database. (C) The upregulated miR-205 in ovarian cancer tissues was confirmed by quantitative polymerase chain reaction. (D) Kaplan-Meier curve for survival rate of patients with high and low miR-205 expression levels. The miR-205 high expression group exhibited shorter survival rates compared with the miR-205 low expression group. ${ }^{*} \mathrm{P}<0.05,{ }^{* *} \mathrm{P}<0.01$. miR, microRNA; TCGA, The Cancer Genome Atlas.

groups. In the survival analysis, the number of patients was 308, with a specified time interval as the endpoint. The Kaplan Meier curve demonstrated that the miR-205 high expression group exhibited shorter survival rates compared with the miR-205 low expression group (Fig. 1D; $\mathrm{P}=0.0210$ ). The result suggests that upregulated miR-205 may be a novel prognostic marker for patients with ovarian cancer.

miR-205 overexpression promotes the proliferation and invasion of ovarian cancer cells. miRNAs exert important functions in tumors via negatively regulating gene expression in a sequence-specific manner (14). Therefore, the function of miR-205 target genes was analyzed by GO analysis. As demonstrated in Fig. 2A, the gene sets of 'regulating cell proliferation' and 'regulation of cell invasion' were highly enriched in the miR-205 target genes, which suggests that miR-205 expression level was positively associated with cell proliferation and invasion. The regulation mechanism and cell experiments were performed in type I epithelial ovarian cancer cells. The results indicated that miR-205 overexpression significantly promoted the proliferation of OVCAR-3 cells, particularly after $48 \mathrm{~h}$ incubation, as compared with control cells (Fig. 2B; $\mathrm{P}<0.01)$. Concurrently, upregulated miR-205 also promoted anchorage-independent growth of OVCAR-3 cells (Fig. 2C; $\mathrm{P}<0.01)$. However, no significant difference was observed between un-transfected and negative control cells. As MMPs-2 and -9 markedly promotes malignant tumor cell invasion, a gelatin zymography assay was performed to detect the activity of MMP-2 and -9. In this experiment, quantitative gray analysis of MMP-2 and -9 expression was performed. The results revealed that there was a difference between the miR-205 overexpression group and the control group (data not shown; $\mathrm{P}=0.0271$ ), particularly for MMP-2. Next, the Transwell assay additionally confirmed that miR-205 overexpression significantly enhanced the invasion ability of ovarian cancer cells compared with the control group (Fig. 3; $\mathrm{P}<0.05$ ). These data suggest that miR-205 promotes ovarian cancer cell proliferation and invasion.

PTEN and SMAD4 are key target genes of miR-205 in ovarian cancer. miRNAs exert important functions in various biological processes via directly suppressing the expression of their target genes. However, for the same miRNAs, target genes and involved pathways are different in different tissues $(15,16)$. To identify key and specific target genes of miR-205 in patients with ovarian cancer, 28 target genes of miR-205 that were associated with proliferation and invasion, and that had multiple data in the miRTarBase database were selected, and a protein-protein interaction (PPI) network was constructed (Fig. 4A). The number of interaction nodes was counted (Fig. 4B), and SMAD4 and PTEN possessed the highest number of interaction nodes within the interaction network of target genes of miR-205. These results confirmed that miR-205 promotes ovarian cancer occurrence, progression and poor prognosis primarily via regulating SMAD4 and PTEN expression.

SMAD4 and PTEN expression levels are downregulated by miR-205 in ovarian cancer. SMAD4 and PTEN are well-known tumor suppressors in multiple types of tumors. The abnormal downregulation of PTEN and SMAD4 protein serves an important role in the malignant transformation of tumors (4). The IHC data were downloaded from the Protein Atlas database. In this database, histological type was not classified. Therefore, the expression of PTEN/SMAD4 in all types ovarian cancer was analyzed. The expression levels of PTEN and SMAD4 in multiple types of ovarian cancer tissues 


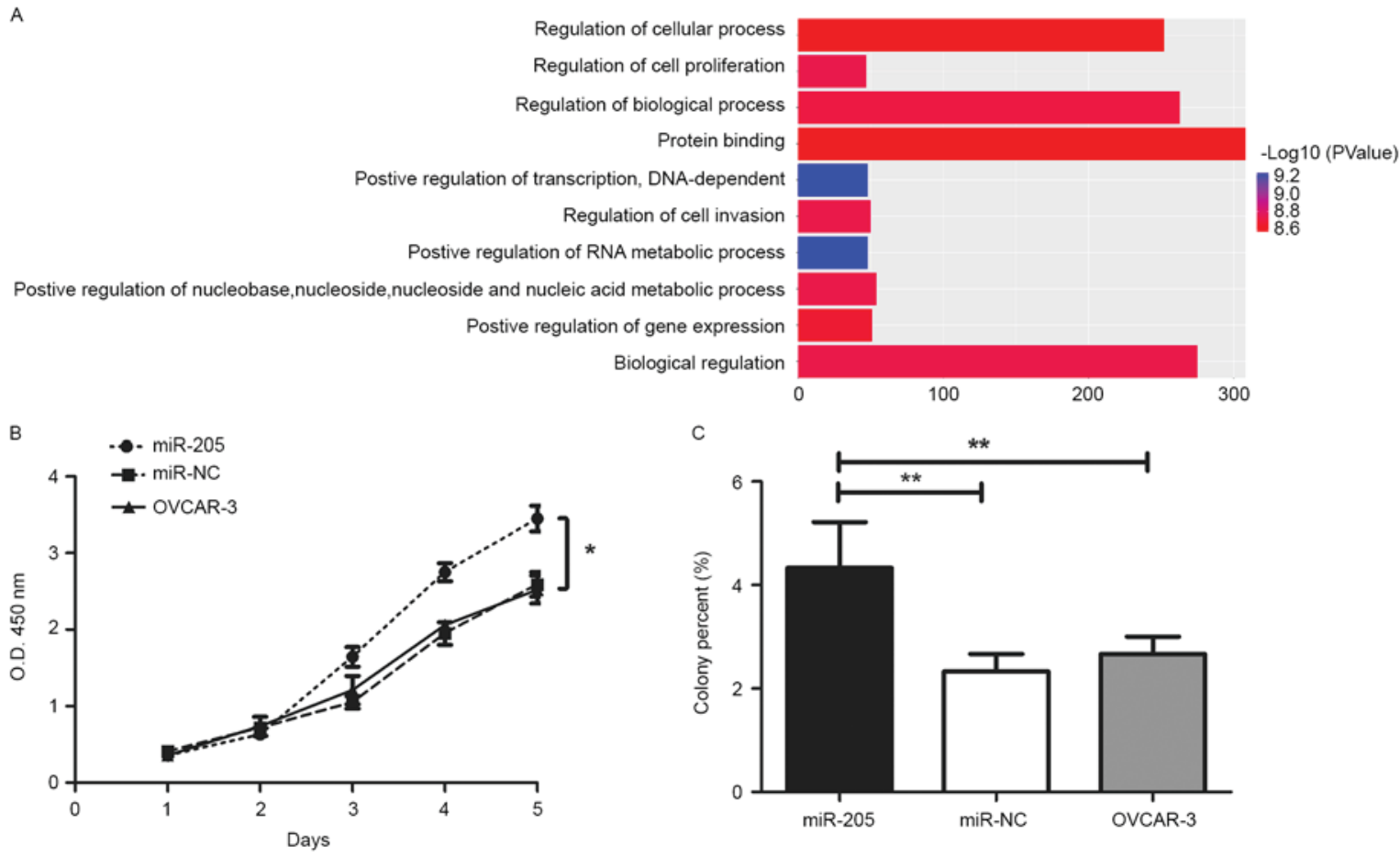

Figure 2. miR-205 promotes proliferation and anchorage-independent growth of ovarian cancer cells. (A) Gene ontology analysis was performed to annotate the functions of the miR-205 target genes involved. 'Cell proliferation', 'invasion' and 'protein binding' were the primary functions of these target genes. (B) miR-205 overexpression significantly enhanced the ability of cell proliferation. (C) miR-205 overexpression significantly enhanced the ability of cell anchorage-independent growth. ${ }^{*} \mathrm{P}<0.05,{ }^{* *} \mathrm{P}<0.01$. miR, microRNA.
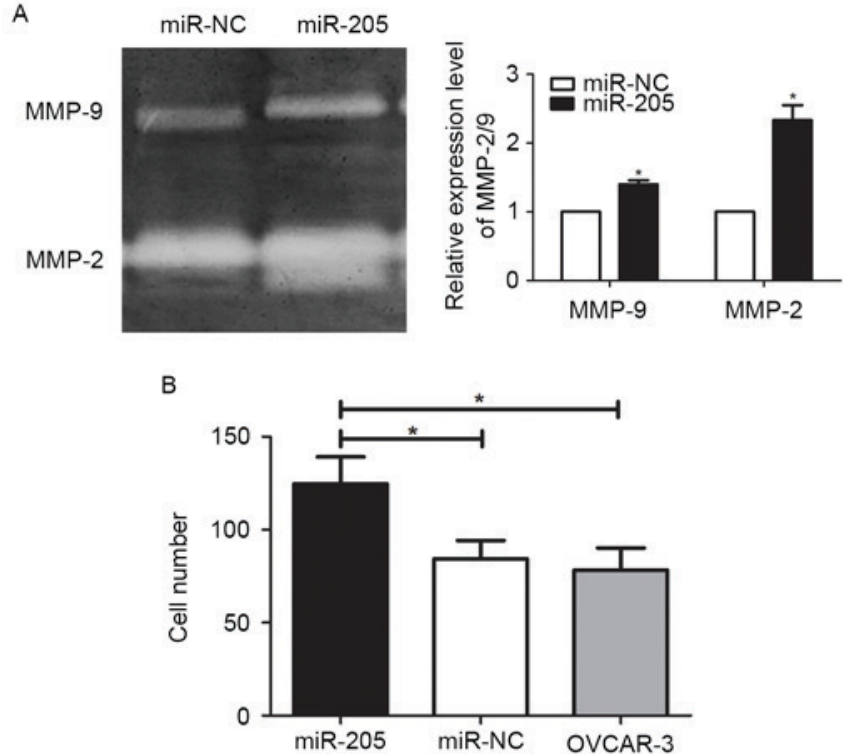

Figure 3. miR-205 enhances the activity of MMP-2 and -9 and promotes ovarian cancer cell invasion. (A) The expression levels of MMP-2 and -9 in OVCAR-3 cells transfected with miR-205 were significantly upregulated compared with the control group. (B) Compared to control group, more cells had migrated through the Transwell membrane in the miR-205 overexpression group, suggesting that miR-205 significantly promoted OVCAR-3 cells invasion. ${ }^{\text {" }} \mathrm{P}<0.05$. miR, microRNA; MMP, matrix metalloproteinase.

were significantly downregulated (Fig. 5A and B; $\mathrm{P}=0.0004$; $\mathrm{P}=0.001$ respectively) compared with normal ovary tissues. In the ovarian cancer tissues, TCGA database was used to analyze the expression of miR-205. However, Fig. 5A and B were used to analyze the expression level of PTEN/SMAD4, not to miR-205. To additionally validate if miR-205 may directly regulate the expression of PTEN and SMAD4, miR-205 mimics was transfected into OVCAR-3 cells and the expression levels of PTEN and SMAD4 were detected by western blot analysis. The results demonstrated that the expression levels of PTEN and SMAD4 were reduced with miR-205 overexpression in OVCAR-3 cells (Fig. 5C), and miR-205 may suppress the expression of PTEN 4 and SMAD in ovarian cancer.

\section{Discussion}

In the present study, the expression level of miR-205 was analyzed in 308 patients. The expression level of PTEN/SMAD4 was analyzed by database, but the expression level regulated by miR-205 was confirmed by cell experiment. In the present study, TCGA data analysis suggested that the expression level of miR-205 was significantly upregulated in ovarian cancer tissues and was markedly correlated with poor prognosis. GO analysis revealed that the target genes of miR-205 were primarily involved in cell proliferation and invasion, and upregulated miR-205 promoted the proliferation and invasion of ovarian cancer cells in vitro. Next, PTEN and SMAD4 were identified as the key targets of miR-205 by PPI networks. The expression level of PTEN and SMAD4 were significantly downregulated in ovarian cancer tissues compared to normal ovary tissues. In vitro, it was additionally confirmed that miR-205 overexpression directly decreased the expression level of PTEN and SMAD4 in OVCAR-3 cells. These results suggested that miR-205 may promote cell proliferation and invasion primarily via suppressing the expression of PTEN/SMAD4. These results 

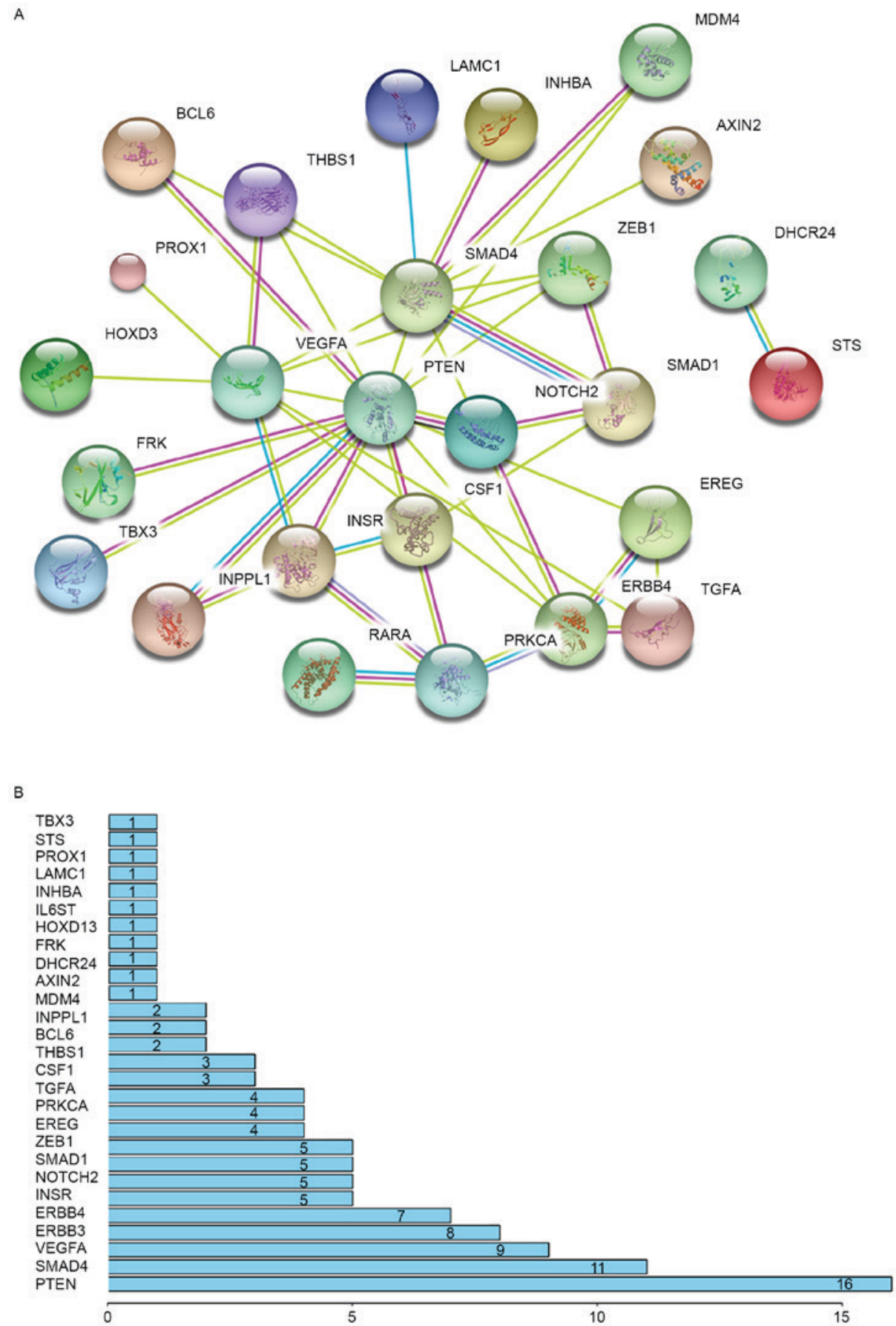

Figure 4. PTEN and SMAD4 are key target genes of miR-205. (A) A protein-protein interaction network was constructed for 28 target genes of miR-205. (B) The node numbers were counted, and PTEN and SMAD4 were at the core of the interaction network. PTEN, phosphatase and tensin homolog; SMAD4, mothers against decapentaplegic homolog 4; miR, microRNA.

assist in providing a potential novel prognostic marker and therapeutic target for ovarian cancer.

Previous work demonstrated that miR-205 was aberrantly expressed in breast cancer, prostate cancer and cutaneous squamous cell carcinoma (16-19). miR-205 is primarily involved in radioresistance, chemotherapeutics resistance, prognosis prediction and cell proliferation $(16,19,20)$. Upregulation of miR-205 under hypoxia promotes epithelial-mesenchymal transition by targeting ASPP2 (21). Inhibition of miR-205 expression using LNA-modified-nucleotide attenuated endometrial cancer cells proliferation in vitro and in vivo (20). miR-205 was expressed in tumors with pathological features recognized as indicators of poor prognosis such as desmoplasia, perineural invasion and infiltrative growth pattern (17). Similarly, miR-205 was also demonstrated to be upregulated in ovarian cancer tissues compared with normal tissues (22), and enhanced the ability of cell invasion and migration by targeting vascular endothelial growth factor and Zinc Finger E-box binding homeobox $1(23,24)$. In the present study, it was initially identified that miR-205 acts as a novel prognostic marker in patients with ovarian cancer, and it was additionally confirmed that miR-205 enhanced the ability of proliferation and anchorage-independent growth of ovarian cancer cells. In addition, it was also verified that miR-205 enhanced 
A

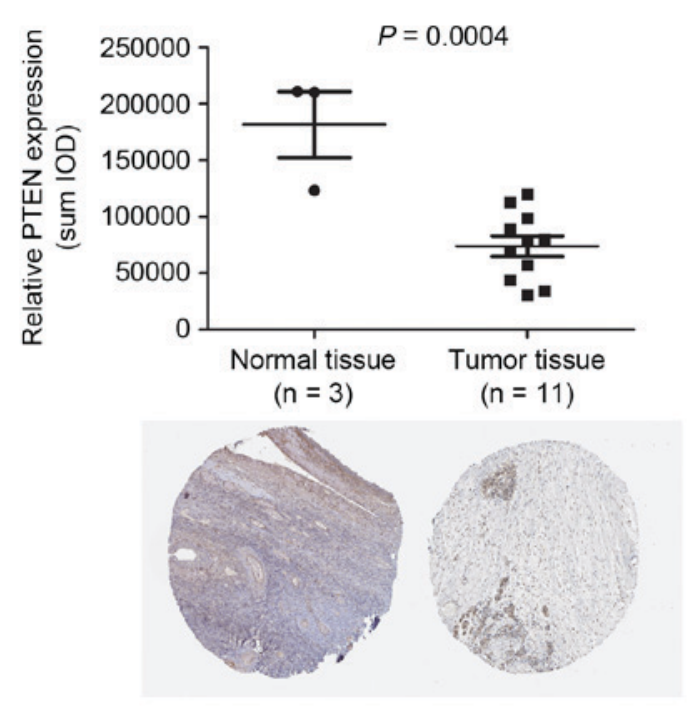

C

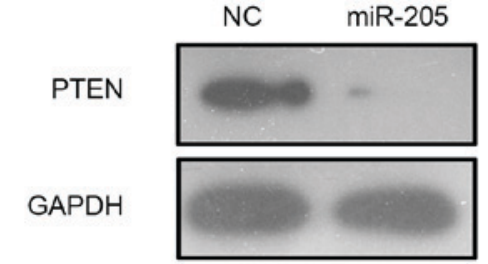

B
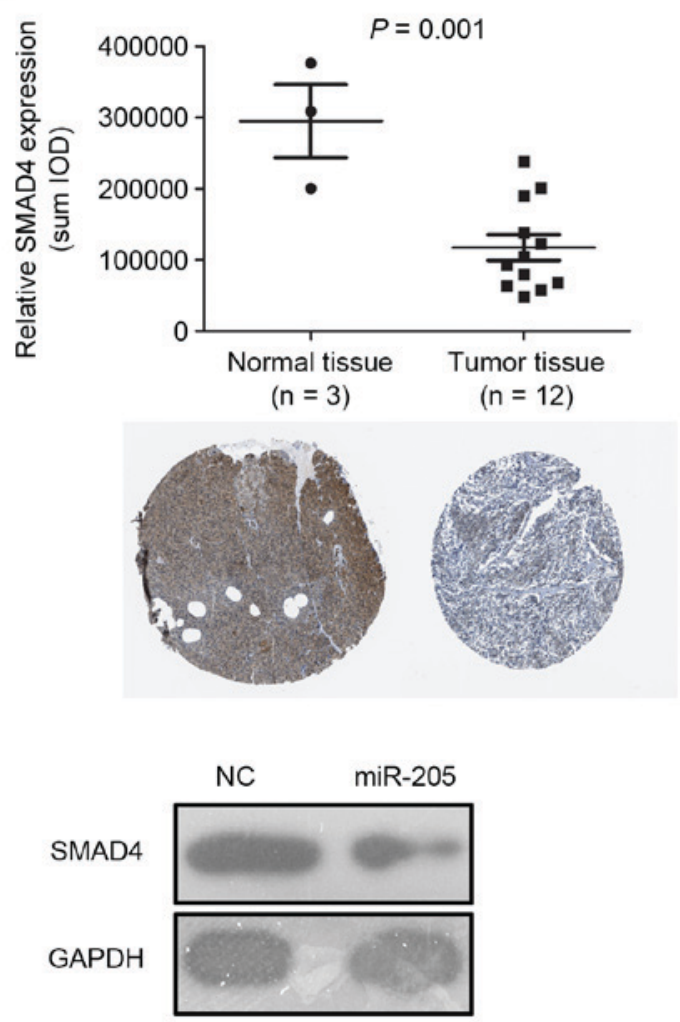

Figure 5. miR-205 suppresses the expression of PTEN and SMAD4 in ovarian cancer cells. The Human Protein Atlas database results revealed that the expression level of (A) PTEN and (B) SMAD4 were downregulated in ovarian cancer tissues compared with normal tissues. (C) Western blotting analysis confirmed that miR-205 inhibited the expression of PTEN and SMAD4 in OVCAR-3 cells. miR, microRNA; PTEN, phosphatase and tensin homolog; SMAD4, mothers against decapentaplegic homolog 4 (magnification, $\mathrm{x} 400$ ).

ovarian cancer cell invasion by regulating the expression of MMP-2/9 in vitro. These data indicate that the most important mechanism by which miR-205 leads to the poor prognosis for ovarian cancer may be primarily via enhancing the ability of cell proliferation and invasion.

miRNAs serve their roles via suppressing the expression of target mRNAs (4). The biological regulation between miRNAs and their targets is complex in vivo (4). The GO analysis results revealed that protein binding was one of the important functions for the miR-205 target genes. PPI analysis was then performed for the target genes of miR-205 associated with cell proliferation and invasion. The results from the PPI analysis revealed that 28 primary target proteins for miR-19a in ovarian cancer interacted and formed an interactive network. Among these 28 target proteins, PTEN and SMAD4 were key node proteins for the interaction between target genes of miR-205 in ovarian cancer. Ma et al (4) identified that PTEN/SMAD4 were target genes of miR-19a and involved in cell proliferation. In the present study, the action of SMAD4 and PTEN as the key ovarian cancer-specific target genes of miR-205 was additionally verified by analysis of the expression levels in tissues and in vitro. The expression levels of PTEN and SMAD4 were significantly downregulated in ovarian cancer tissues compared with the normal tissue, and upregulated miR-205 markedly suppressed the expression level of PTEN and SMAD4. We hypothesized that miR-205 coordinately regulated the expression level and associated signaling pathways of PTEN and SMAD4 to affect the different phases of ovarian cancer progression. In fact, the expression level of PTEN/SMAD4 may also be regulated by certain other miRNAs in other types of tumor, such as miR-19a (4). However, in ovarian cancer, other miRNAs that regulated the expression of PTEN/SMAD4 were not identified. In the present study, it was initially demonstrated that miR-205 may regulate the expression level of PTEN/SMAD4, and contribute to tumor malignant phenotype. Additionally, Li et al (25) also identified that miR-205 is an oncogene in ovarian cancer via suppressing the expression of PTEN/SMAD4, supporting the conclusions of the present study. As luciferase reporter gene assays are the primary method to identify target genes of miRNA (7), the results of the present study only suggest that miR-205 may regulate the expression level of PTEN and SMAD4. The present study aimed to confirm whether miR-205 may affect the malignant phenotype of ovarian cancer cells via regulating the expression level of PTEN and SMAD4, irrespective of the direct or indirect interaction. Direct interactions between PTEM/SMAD4 and miR-205 will be confirmed in future studies. Future studies also require larger samples to confirm the preliminary results of the present study.

To conclude, miR-205 may represent a novel potential prognostic biomarker for ovarian cancer and miR-205 promoted ovarian cancer cell proliferation and invasion, primarily by targeting PTEN and SMAD4. To the best of our knowledge, the present study is the first to demonstrate the prognosis predictive value of miR-205 for patients with 
ovarian cancer, and provide a novel direction for ovarian cancer treatment.

\section{Acknowledgements}

Not applicable.

\section{Funding}

This study was supported by The National Natural Science Fund (grant no. 81701532), The Basic Research of Natural Science of Shaanxi Province (grant no. 2014JQ2-8047) and The Clinical Research Fund of the First Affiliated Hospital of Xi'an Jiaotong University (grant no. XJTU1ARCH2014-029).

\section{Availability of data and materials}

The analyzed data sets generated during the study are available from the corresponding author, on reasonable request.

\section{Author contributions}

PC, AL and LZ designed the present study. PC and AJ performed the research and analyzed the data. PC, AL and LZ wrote the manuscript. The final version of the manuscript has been read and approved by all authors.

\section{Ethics approval and consent to participate}

The present study was approved by the Research Ethics Boards of Binzhou Medical University and Yantai Affiliated Hospital. Written informed consent was obtained from each patient.

\section{Consent for publication}

Not applicable.

\section{Competing interests}

The authors declare that they have no competing interests.

\section{References}

1. Webb PM and Jordan SJ: Epidemiology of epithelial ovarian cancer. Best Pract Res Clin Obstet Gynaecol 41: 3-14, 2017.

2. Mahdian-Shakib A, Dorostkar R, Tat M, Hashemzadeh MS and Saidi N: Differential role of microRNAs in prognosis, diagnosis, and therapy of ovarian cancer. Biomed Pharmacother 84: 592-600, 2016.

3. Yu G, Jia Z and Dou Z: miR-24-3p regulates bladder cancer cell proliferation, migration, invasion and autophagy by targeting DEDD. Oncol Rep 37: 1123-1131, 2017.

4. Ma Q, Peng Z, Wang L, Li Y, Wang K, Zheng J, Liang Z and Liu T: miR-19a correlates with poor prognosis of clear cell renal cell carcinoma patients via promoting cell proliferation and suppressing PTEN/SMAD4 expression. Int J Oncol 49: 2589-2599, 2016.

5. Fu X, Li Y, Alvero A, Li J, Wu Q, Xiao Q, Peng Y, Hu Y, Li X, Yan W, et al: MicroRNA-222-3p/GNAI2/AKT axis inhibits epithelial ovarian cancer cell growth and associates with good overall survival. Oncotarget 7: 80633-80654, 2016.

6. Jeong JY, Kang H, Kim TH, Kim G, Heo JH, Kwon AY, Kim S, Jung SG and An HJ: MicroRNA-136 inhibits cancer stem cell activity and enhances the anti-tumor effect of paclitaxel against chemoresistant ovarian cancer cells by targeting Notch3. Cancer Lett 386: 168-178, 2017.
7. Tian S, Zhang M, Chen X, Liu Y and Lou G: MicroRNA-595 sensitizes ovarian cancer cells to cisplatin by targeting ABCB1. Oncotarget 7: 87091-87099, 2016.

8. Zhou F, Chen J and Wang H: MicroRNA-298 inhibits malignant phenotypes of epithelial ovarian cancer by regulating the expression of EZH2. Oncol Lett 12: 3926-3932, 2016.

9. $\mathrm{Hu} \mathrm{K}$ and Liang M: Upregulated microRNA-224 promotes ovarian cancer cell proliferation by targeting KLLN. In Vitro Cell Dev Biol Anim 53: 149-156, 2017.

10. Liang T, Li L, Cheng Y, Ren C and Zhang G: MicroRNA-194 promotes the growth, migration, and invasion of ovarian carcinoma cells by targeting protein tyrosine phosphatase nonreceptor type 12. Onco Targets Ther 9: 4307-4315, 2016.

11. Wu AH, Huang YL, Zhang LZ, Tian G, Liao QZ and Chen SL: MiR-572 prompted cell proliferation of human ovarian cancer cells by suppressing PPP2R2C expression. Biomed Pharmacother 77: 92-97, 2016.

12. Szklarczyk D, Morris JH, Cook H, Kuhn M, Wyder S, Simonovic M, Santos A, Doncheva NT, Roth A, Bork P, et al: The STRING database in 2017: Quality-controlled protein-protein association networks, made broadly accessible. Nucleic Acids Res 45(D1): D362-D368, 2017.

13. Marko TA, Shamsan GA, Edwards EN, Hazelton PE, Rathe SK, Cornax I, Overn PR, Varshney J, Diessner BJ, Moriarity BS, et al: Slit-Robo GTPase-activating protein 2 as a metastasis suppressor in osteosarcoma. Sci Rep 6: 39059, 2016.

14. Livak KJ and Schmittgen TD: Analysis of relative gene expression data using real-time quantitative PCR and the 2(-Delta Delta C(T)) method. Methods 25: 402-408, 2001.

15. Ito N, Sakai A, Miyake N, Maruyama M, Iwasaki H, Miyake K, Okada T, Sakamoto A and Suzuki H: miR-15b mediates oxaliplatin-induced chronic neuropathic pain through BACE1 downregulation. Br J Pharmacol 174: 386-395, 2017.

16. Hu Y, Qiu Y, Yagüe E, Ji W, Liu J and Zhang J: miRNA-205 targets VEGFA and FGF2 and regulates resistance to chemotherapeutics in breast cancer. Cell Death Dis 7: e2291, 2016.

17. Cañueto J, Cardeñoso-Álvarez E, García-Hernández JL, Galindo-Villardón P, Vicente-Galindo P, Vicente-Villardón JL, Alonso-López D, De Las Rivas J, Valero J, Moyano-Sanz E, et al: MicroRNA (miR)-203 and miR-205 expression patterns identify subgroups of prognosis in cutaneous squamous cell carcinoma. Br J Dermatol 177: 168-178, 2017.

18. Osipov ID, Zaporozhchenko IA, Bondar AA, Zaripov MM, Voytsitskiy VE, Vlassov VV, Laktionov PP and Morozkin ES: Cell-free miRNA-141 and miRNA-205 as prostate cancer biomarkers. Adv Exp Med Biol 924: 9-12, 2016.

19. Pan F, Mao H, Bu F, Tong X, Li J, Zhang S, Liu X, Wang L, Wu L, Chen R, et al: Sp1-mediated transcriptional activation of miR-205 promotes radioresistance in esophageal squamous cell carcinoma. Oncotarget 8: 5735-5752, 2017.

20. Torres A, Kozak J, Korolczuk A, Rycak D, Wdowiak P, Maciejewski R and Torres K: Locked nucleic acid-inhibitor of miR-205 decreases endometrial cancer cells proliferation in vitro and in vivo. Oncotarget 7: 73651-73663, 2016.

21. Wang X, Yu M, Zhao K, He M, Ge W, Sun Y, Wang Y, Sun H and Hu Y: Upregulation of MiR-205 under hypoxia promotes epithelial-mesenchymal transition by targeting ASPP2. Cell Death Dis 7: e2517, 2016.

22. Iorio MV, Visone R, Di Leva G, Donati V, Petrocca F, Casalini P, Taccioli C, Volinia S, Liu CG, Alder H, et al: MicroRNA signatures in human ovarian cancer. Cancer Res 67: 8699-8707, 2007.

23. Niu K, Shen W, Zhang Y, Zhao Y and Lu Y: MiR-205 promotes motility of ovarian cancer cells via targeting ZEB1. Gene 574: 330-336, 2015.

24. Li J, Li L, Li Z, Gong G, Chen P, Liu H, Wang J, Liu Y and Wu X: The role of miR-205 in the VEGF-mediated promotion of human ovarian cancer cell invasion. Gynecol Oncol 137: 125-133, 2015.

25. Li J, Hu K, Gong G, Zhu D, Wang Y, Liu H and Wu X. Upregulation of MiR-205 transcriptionally suppresses SMAD4 and PTEN and contributes to human ovarian cancer progression. Sci Rep 7: 41330, 2017.

This work is licensed under a Creative Commons Attribution-NonCommercial-NoDerivatives 4.0 International (CC BY-NC-ND 4.0) License. 